\title{
Factores que no propenden la internacionalización de las pequeñas y medianas empresas en Puerto Rico
}

\author{
Edgar J. Maíz Vázquez / emaiz@br.inter.edu \\ Universidad Interamericana de Puerto Rico \\ Recinto de Barranquitas
}

\section{RESUMEN:}

El objetivo de este estudio es determinar cómo inciden la orientación empresarial y el aprendizaje de la organización (factores internos) y el ambiente/industria y competencia (factores externos) en la internacionalización de las pequeñas y medianas empresas (PYMES) en Puerto Rico. Sólo el ambiente mostró una relación estadísticamente significativa con las ventas internacionales. Los hallazgos sugieren que las PYMES exportadoras en Puerto Rico son empresas tradicionales que presentan una orientación empresarial dirigida primordialmente a la adquisición de una estabilidad y de una solidez dentro del mercado nacional, para luego considerar exportar.

Palabras clave: pequeñas y medianas empresas, empresarismo, internacionalización, exportación.

\section{ABSTRACT:}

The objective of this study is to determine the influence of the entrepreneurial orientation and the organizational learning (internal factors) and the environment/ industry and competition (external factors) in the internationalization of small and medium-sized enterprises (SME) in Puerto Rico. Only the environment showed statistically significant relation (negative coefficient) with the international sales. The findings suggest that the exporting SME in Puerto Rico are traditional businesses that show an entrepreneurial orientation directed primarily to the acquisition of stability in the domestic market and then consider export.

Keywords: small and medium-sized enterprises, entrepreneurship, internationalization, export. 


\section{INTRODUCCIÓN}

De acuerdo con la Organización para la Cooperación y Desarrollo Económico (OCDE), las pequeñas y medianas empresas (PYMES) constituyen sobre el $95 \%$ de las empresas, generan dos terceras partes de los empleos del sector privado y son la mayor fuente de empleos nuevos, dependiendo del país (2005). La OCDE estima que las PYMES contribuyen entre el $25 \%$ y el $35 \%$ de las exportaciones de manufactura a nivel mundial. A pesar de la creciente importancia de las PYMES en las economías nacionales, estudios indican que aproximadamente $20 \%$ de las PYMES nuevas no sobreviven más allá de un segundo año de operaciones (OCDE, 2005). Según Global Entrepreneurship Monitor (GEM, 2007), las cifras de las PYMES que logran exportar su producto y/o servicio son aún menores. La literatura académica ha realizado esfuerzos para examinar las PYMES que sobreviven y logran internacionalizarse (Etemad, 2004).

GEM clasifica a Puerto Rico como un país de alto ingreso. Entre los 23 países clasificados como de alto ingreso, Puerto Rico ocupó el tercer lugar como el país de menor actividad empresarial general después de Bélgica y Francia (GEM, 2007). El GEM puede considerarse como una herramienta útil para establecer un perfil del empresarismo y la actividad empresarial en Puerto Rico, pero no presenta resultados de la influencia de los mercados extranjeros en las empresas en Puerto Rico. En Puerto Rico, los estudios recientes sobre la internacionalización de las PYMES se han concentrado en estudios de casos (Orengo-Serra, 2007). Con relación al tema de la internacionalización de las PYMES, tanto la literatura académica como las iniciativas de organizaciones y consorcios internacionales analizan los aspectos y/o factores que permiten identificar problemas y prácticas comunes.

Esta investigación busca determinar las propiedades, las características o los rasgos de la internacionalización de las PYMES en Puerto Rico. Siguiendo esa misma línea de análisis, el objetivo de la investigación es examinar cómo inciden los aspectos o factores internos y externos de las PYMES en Puerto Rico en la internacionalización de las mismas. 


\section{MARCO TEÓRICO E HIPÓTESIS}

Mucha de la literatura académica (Coviello \& McAuely, 1999), así como textos de comercio internacional (Hill, 2003), gerencia (Rodrigues, 2001; Cullen \& Parboteeah, 2005) y mercadeo internacional (Cateora \& Graham, 2007; Keegan, 1999) presentan y aceptan la internacionalización de las empresas como un proceso secuencial en el que las empresas aumentan su compromiso con operaciones internacionales según adquieren conocimiento sobre los mercados internacionales (e.g., culturas, idiomas, prácticas comerciales extranjeras, etc.). Muchas empresas (pequeñas, medianas y grandes) encuentran que el proceso de internacionalización planificado por etapas es una herramienta de análisis adecuada que ayuda a definir su situación estratégica (Cullen \& Parboteeah, 2005). Aplicar ese modelo de internacionalización por etapas permite a las compañías minimizar su exposición al riesgo y desarrollar de forma gradual su pericia internacional (Johanson \& Vahlne, 1990).

A pesar de la aceptación de los modelos de etapas graduales, éstos han sido criticados por no poder explicar completamente la internacionalización actual de las pequeñas empresas (Andersen, 1993; Andersson, 2000; Andersson, Gabrielsson \& Wictor, 2004; Oviatt \& McDougall, 1997). Específicamente, se ha encontrado que muchas pequeñas y medianas empresas en diferentes partes del mundo parecen no seguir el patrón tradicional de internacionalización propuesto por la teoría de etapas y han comenzado a exportar con solamente varios años de haberse establecido (Moen \& Servais, 2002; Rennie, 1993). Dicho fenómeno ha sido identificado en la literatura como "born global", el cual plantea la tesis de que muchas empresas logran internacionalizarse, o al menos adquieren cierto grado de internacionalización, al poco tiempo de comenzar operaciones.

El fenómeno "born global" se ha evidenciado en empresas de países de habla hispana como España (Rialp, Rialp, Urbano \& Vaillant, 2005) así como en países pequeños como Dinamarca, Noruega (Moen \& Servais, 2002) y Suecia (Andersson \& Wictor, 2003). También hay evidencias en Australia (Rennie, 1993) y en islas del Pacífico, cuyas economías se encuentran en etapa de desarrollo como 
la de Nueva Zelanda (Chetty \& Campbell, 2004). Básicamente, el criterio para la clasificación del fenómeno "born global" ha sido que las empresas alcancen un grado de internacionalización con un mínimo $25 \%$ de las ventas totales provenientes del extranjero (Andersson \& Wictor, 2003; Moen \& Servais, 2002), aunque el tiempo para alcanzar dicha proporción fluctúa entre dos y seis años después de comenzar operaciones (Coviello \& Munro, 1995).

Dado lo anterior, han surgido estudios que buscan explicar la variabilidad, si alguna, de la internacionalización de las PYMES. La literatura académica reciente ofrece varios marcos teóricos (Ruzzier, Hisrich, \& Antoncic, 2006). Etemad (2004) presenta uno de los más delineados y atemperado a los últimos hallazgos, en el que conceptualiza y divide los factores que median o propenden la internacionalización de las PYMES como fuerzas que impulsan (factores internos) y fuerzas que halan (factores externos) a la internacionalización.

La identificación o clasificación de las variables independientes en factores internos y externos facilita un análisis que abona a la explicación de los aspectos estratégicos, si alguna, sobre la internacionalización de las PYMES. Desde un punto de vista de gerencia estratégica, los factores internos son considerados aspectos propios de las empresas que representan fortalezas y debilidades que ayudan al desarrollo de estrategias y tácticas en las mismas (David, 2009). Entre los factores internos, Etemad (2004) incluye las características y las visiones de los dueños y/o gerentes, los que en la literatura y estudios recientes se identifican como la orientación de la empresa u orientación empresarial (Todd, 2006; De Clercq, Sapienza \& Crijns, 2005).

La orientación empresarial del dueño ha sido utilizada en estudios como variable explicativa de la expansión geográfica de la actividad comercial de las pequeñas y medianas empresas (Andersson \& Wictor, 2003). Dicha orientación ha sido conceptualizada y medida en términos de grados de innovación, toma de decisiones riesgosas y actividades pro-activas desempeñadas por las empresas (Covin \& Slevin, 1991). Estudios recientes han encontrado una relación positiva entre altos niveles de orientación empresarial y el grado de internacionalización (Menguzzato-Boulard \& Sanchez-Peinado, 2007; Todd, 2006), y el intento de internacionalización de las pequeñas y medianas empresas (De Clercq et al., 2005). 
Otra variable independiente (y factor interno) sugerida por Etemad (2004) es el aprendizaje de la organización. Éste ha sido definido como los esfuerzos de la organización dirigida a desarrollar conocimientos sobre las actividades comerciales internacionales (De Clercq et al., 2005), que pueden vincularse a las redes comerciales (Musteen, 2006). El nivel de aprendizaje organizacional ha sido conceptualizado como el proceso o el esfuerzo de explotar el conocimiento existente (De Clercq et al., 2005) y explorar o asimilar el conocimiento nuevo dentro de la base de conocimiento de la organización (Autio, Sapienza \& Almeida, 2000). Estudios empíricos han encontrado que la adquisición y explotación de conocimiento están asociados positivamente con el crecimiento de ventas internacionales (Autio et al., 2000) así como con el intento de la internacionalización de las pequeñas y medianas empresas (De Clercq et al., 2005).

De acuerdo con la literatura, las variables orientación empresarial y los esfuerzos de aprendizaje de una organización se consideran factores propios de una empresa, los cuales pueden representar fortalezas internas que ayudan a tomar ventaja de oportunidades que ofrecen factores externos como es el ambiente/industria. A base de los planteamientos teóricos y hallazgos empíricos discutidos anteriormente, se plantearon las siguientes hipótesis:

$\mathbf{H}_{1}$ : La orientación empresarial está relacionada positivamente con la internacionalización de las PYMES en Puerto Rico.

$\mathbf{H}_{2}$ : El esfuerzo de aprendizaje de la organización está relacionado positivamente con la internacionalización de las PYMES en Puerto Rico.

El marco teórico de Etemad (2004) también considera factores externos como el ambiente/industria y la competencia que halan a las empresas a la internacionalización. Desde un punto de vista de gerencia estratégica, el ambiente/industria y la competencia son considerados factores externos que pueden ofrecer oportunidades y/o amenazas al desarrollo de las empresas (David, 2009). El ambiente de las organizaciones se ha categorizado en tres dimensiones: dinamismo, hostilidad y complejidad (Covin \& Slevin, 1989, 1991; 
Dess \& Beard, 1984; Miller \& Friesen, 1982). El impacto del ambiente externo en el desempeño de las empresas se ha evaluado de muchas maneras. Estudios empíricos han determinado que existe una relación positiva entre el dinamismo del ambiente y la introducción de productos nuevos de las empresas (Zahra, 1993), así como una relación positiva con la toma de riesgos en la inversión de recursos para la entrada a mercados extranjeros (Rasheed, 2005). No obstante, también se ha encontrado que existe una relación negativa entre la hostilidad del ambiente y el grado en que una empresa decide adoptar decisiones riesgosas y actividades proactivas (i.e., orientación empresarial) (Miles, Arnold \& Thompson, 1993). Estudios recientes resaltan que el dinamismo del ambiente externo modera (moderate) el grado de internacionalización (Todd, 2006) y el desempeño de las actividades de exportación de las PYMES (French, 2006).

Los estudios también han incluido la competencia como parte del ambiente externo que influye en el comportamiento de las empresas (Covin \& Slevin, 1989). Incluso, existen estudios que proponen el nivel de competencia como una variable independiente del ambiente externo y que éste influye grandemente en la percepción de los empresarios y sus actuaciones (Dodge, Fullerton \& Robbins, 1994).

De acuerdo con la literatura, las variables ambiente y competencia de una organización son considerados factores externos de una empresa, los cuales pueden influir positiva o negativamente en las percepciones de los empresarios con respecto a las estrategias y operaciones que siguen sus empresas. A base de los planteamientos teóricos y hallazgos empíricos discutidos, se plantearon las siguientes hipótesis:

$\mathbf{H}_{3}$ : El ambiente/industria está relacionado positivamente con la internacionalización de las PYMES en Puerto Rico.

$\mathbf{H}_{4}$ : La competencia es relacionada positivamente con la internacionalización de las PYMES en Puerto Rico. 


\section{Metodología}

\section{MUESTRA Y COLECCIÓN DE DATOS}

La muestra del estudio fue tomada del Export Catalogue and Interactive Directory (ECID), un directorio de empresas exportadoras desarrollado por la Compañía de Comercio y Exportación de Puerto Rico, una corporación pública bajo el Departamento del Desarrollo Económico y Comercio del Gobierno de Puerto Rico. Dado el objetivo del estudio, la muestra sólo incluyó empresas independientes (no subsidiarias de empresas más grandes) con menos de 500 empleados, características consistentes con la definición de pequeñas y medianas empresas aceptadas y utilizadas en estudios previos (OECD, 2005). Dichas empresas debían tener sus oficinas centrales en Puerto Rico y tenían que estar exportando activamente. En mayo de 2008 se identificaron 120 empresas de diversas industrias (manufactura, servicio, detallistas y mayoristas) que cumplían con las características antes mencionadas. Se les envió un cuestionario a todos los dueños o gerentes de ventas internacionales de estas empresas, y se recibieron 71 cuestionarios debidamente contestados, lo que representa un $59 \%$ de tasa de respuesta.

\section{Medición}

Se utilizó un cuestionario como instrumento de medición, mediante el cual se buscó evaluar la forma en que inciden la orientación empresarial, el esfuerzo de aprendizaje organizacional (factores internos de la empresa), el ambiente/industria y la competencia (factores externos de la empresa) en la internacionalización de las PYMES en Puerto Rico. Se especificó un modelo (ecuación de regresión) donde la variable dependiente ventas internacionales (INTSALES) será una función de las variables independientes (orientación empresarial (ENTREORIENT), ambiente (ENVIRONMENT), esfuerzo de aprendizaje organizacional (ORGLEARNING) y competencia (COMPETENCE) más un término de error, como se establece en la siguiente ecuación:

INTSALES $=\beta_{0}+\beta_{1}$ ENTRORIENT $+\beta_{2}$ ENVIRONMENT $+\beta_{3}$ ORGLEARNING $+\beta_{4}$ COMPETENCE + Error 
La variable dependiente internacionalización fue medida como la proporción o por ciento de las ventas/ingreso totales que provienen de mercados extranjeros. Dicha medida es consistente con estudios recientes (Ogunmokun \& Ng, 2004; Todd, 2006; Wolff \& Pett, 2000).

La variable independiente orientación empresarial, considerada como factor interno, fue medida utilizando la escala de 9 ítems desarrollada por Covin y Slevin (1989). Estos autores presentan la escala orientación empresarial como un estilo empresarial estratégico que incluye tres subdimensiones: propensión a la innovación, proactividad y toma de decisiones. Para determinar si los ítems podían ser combinados en una sola escala de orientación empresarial, es decir, evaluar la dimensionalidad o validez factorial, se realizó un análisis de factores. Las subdimensiones mostraron cargas (loadings) sobre .70 (cargas promedio de $.75, .65$ y .79 , respectivamente), lo que indica que era apropiado combinar los ítems en una sola escala. Esta escala de orientación empresarial de 1 a 7 , presentó un promedio de 4.47 y un rango de respuesta de 2.44 a 6.67 .

La variable independiente ambiente/industria, considerada como factor externo, fue medida utilizando la escala de 12 ítems desarrollada por Miller y Friesen (1982). Esta escala se utiliza compuesta de tres subdimensiones: el dinamismo, la hostilidad y la complejidad (heterogeneidad). Los encuestados debían responder en una escala Likert de 1 a 7, donde cuanto mayor es el número, más dinámico, hostil y complejo era el ambiente. Esta escala presentó una medida de confiabilidad (coeficiente Alpha de Cronbach) de .684, que es marginalmente menor al límite aceptado (.70) para concluir que las escalas de un instrumento de medición son adecuadamente confiables en términos de su consistencia interna (Nunnally, 1978). No obstante, para estudios exploratorios puede aceptarse hasta un .60 como medidas de consistencia internas confiables (Hair, Anderson, Tatham \& Black, 1998). Esta escala de ambiente como escala unidimensional, presentó un promedio de 3.73 y un rango de respuesta de 2.00 a 6.00 .

La otra variable independiente considerada como factor externo, competencia, fue medida utilizando la escala de 6 ítems para medir la intensidad competitiva desarrollada por Jaworski y Kohli 
(1993). Los encuestados debían responder en una escala Likert de 1 a 7 , donde cuanto mayor es el número, mayor la intensidad competitiva. En un análisis de factores, los ítems presentaron una carga (loadings) promedio de .62, lo que indica que era apropiado combinar dicho ítems en una sola escala. Esta escala presentó una medida de confiabilidad (coeficiente Alpha de Cronbach) de .694. Esta escala de competencia o intensidad competitiva presentó un promedio de 4.17 y un rango de respuesta de 1.67 a 6.50 .

La otra variable independiente considerada como factor interno de la empresa, esfuerzo de aprendizaje organizacional, se midió con la escala de 12 ítems desarrollada por De Clercq, Sapienza \& Crijns (2005). De Clercq et al., presentan la escala como una medida de esfuerzos de aprendizaje organizacional en mercados extranjeros compuesta por dos subescalas: ítems que se enfocan en los esfuerzos para las actividades internacionales generales e ítems que se enfocan en factores específicos en el mercado extranjero más importante. Esta escala presentó una medida de confiabilidad (coeficiente Alpha de Cronbach) de .887, mostrando ser una escala confiable en términos de su consistencia interna. En una escala de 1 a 7 , presentó un promedio de 3.99 y un rango de respuesta de 1.08 a 7.00 .

Además del grado de internacionalización de las empresas, el presente estudio también ausculta otros aspectos que ofrezcan un perfil o que expliquen patrones o procesos de la internacionalización de las PYMES en Puerto Rico. Para dichos fines, el cuestionario proveyó preguntas sobre la información general de la empresa, incluyendo cuánto tiempo hace que las empresas operan y exportan. Igualmente, se analizó el modo como las empresas entran o atienden su mercado extranjero, así como los países o el área geográfica destinatarias de sus exportaciones. Estos datos permitieron estudiar la vigencia de la teoría de internacionalización por etapas o secuencial.

El cuestionario también auscultó datos sobre las personas encuestadas, específicamente: edad, nivel de educación, idiomas (además del español) que habla o lee, así como la frecuencia con la que viaja a los mercados extranjeros. También se indagó sobre los años de experiencia en la industria y los años que trabajan los encuestados en el comercio internacional. Igualmente, se realizó un escrutinio de los formatos de adiestramientos en comercio internacional que habían 
recibido los encuestados. Todos estos datos ofrecen información relevante para delinear un perfil de los encuestados en el estudio, que son dueños, ejecutivos y personal gerencial que trabaja las ventas o los clientes fuera de Puerto Rico en sus respectivas empresas.

\section{Resultados}

En la Tabla 1 se presenta un resumen de las estadísticas descriptivas de los datos demográficos de las empresas y sus dueños o gerentes de ventas internacionales.

Tabla 1: Resumen de las estadísticas descriptivas de los datos demográficos de las empresas y los encuestados.

\begin{tabular}{|c|c|c|c|}
\hline Datos demográficos & Media & Mediana & Moda \\
\hline Años de operación & 22.86 & 18 & $21-50$ \\
\hline $\begin{array}{l}\text { Años en el comercio } \\
\text { internacional }\end{array}$ & 9.33 & 7 & $3-5$ \\
\hline Número de empleados & 71.96 & 32 & $1-25$ \\
\hline Ventas totales & $\$ 4.37$ millones & $\$ 3$ millones & $>$ >10millones \\
\hline Ventas internacionales & $23.63 \%$ & $15 \%$ & menos de $10 \%$ \\
\hline $\begin{array}{l}\text { Número de países a los } \\
\text { que exportan }\end{array}$ & 5.73 & 3 & $1-4$ \\
\hline $\begin{array}{l}\text { Regiones destinatarias } \\
\text { de las exportaciones }\end{array}$ & -- & --- & Área del Caribe \\
\hline Edad $^{a}$ & 42.68 & 41 & $35-44$ \\
\hline Nivel de educación ${ }^{a}$ & 17.31 & 16 & Bachillerato \\
\hline Frecuencia de viajes al año ${ }^{a}$ & 2.13 & 2 & $>2$ \\
\hline $\begin{array}{l}\text { Años de experiencia en } \\
\text { la industria }\end{array}$ & 13.8 & 14 & 10 \\
\hline $\begin{array}{l}\text { Años de experiencia en el } \\
\text { comercio internacionala }\end{array}$ & 9.5 & 6 & 5 \\
\hline $\begin{array}{l}\text { Adiestramiento en el } \\
\text { comercio internacional }^{\mathrm{a}}\end{array}$ & -- & -- & $\begin{array}{l}\text { Adiestramiento } \\
\text { en el trabajo }\end{array}$ \\
\hline
\end{tabular}

${ }^{a}$ Estos datos demográficos pertenecen a los individuos que contestaron el cuestionario. 
La interpretación de los datos presentados en la Tabla 1, nos ayuda a delinear un perfil de las empresas encuestadas. Por ejemplo, las empresas encuestadas reflejaron un promedio de aproximadamente 23 años de operación y 9 años en el comercio internacional. Se nota una diferencia significativa, la cual indica que las empresas encuestadas comienzan a exportar un número considerable de años después de comenzar operaciones. Por otro lado, las empresas encuestadas presentaron un promedio de $23.67 \%$ de ventas internacionales, aunque la categoría que más frecuencia presentó fue menos de $10 \%$. En cuanto a las personas encuestadas, éstas reflejaron tener experiencia en su industria y en el comercio internacional donde presentaron promedios de aproximadamente 14 años y 10 años respectivamente. Éstas promediaron más de 17 años de estudios lo que representa más que un bachillerato universitario (representado por 16 años).

Las relaciones entre las variables independientes y dependientes planteadas en las hipótesis de trabajo se evaluaron a través del análisis de regresión múltiple. La Tabla 2 presenta la ecuación y los resultados de dicho análisis.

Tabla 2: Resumen de los resultados del análisis de regresión múltiple para evaluar las hipótesis.

$$
\begin{aligned}
\text { INTSALES }= & \beta_{0}+\beta_{1} \text { ENTRORIENT }+\beta_{2} \text { ENVIRONMENT }+\beta_{3} \text { ORGLEARNING } \\
& +\beta_{4} \text { COMPETENCE }+ \text { Error }
\end{aligned}
$$

\begin{tabular}{|c|c|c|c|c|c|c|}
\hline $\begin{array}{l}\text { Variable } \\
\text { independiente }\end{array}$ & $\begin{array}{l}\text { Variable } \\
\text { dependiente }\end{array}$ & $\mathrm{R}^{2}$ & $\beta$ & $\begin{array}{l}\text { Error } \\
\text { estándar }\end{array}$ & t-value & $p$-value \\
\hline Constante & $\begin{array}{l}\text { Ventas } \\
\text { internacionales }\end{array}$ & .071 & .250 & .180 & 1.387 & .170 \\
\hline $\begin{array}{l}\text { Orientación } \\
\text { empresarial }\end{array}$ & & & .004 & .003 & 1.174 & 245 \\
\hline Ambiente & & & -.005 & .003 & -1.923 & $.059^{*}$ \\
\hline $\begin{array}{l}\text { Esfuerzo de } \\
\text { aprendizaje }\end{array}$ & & & & & & \\
\hline organizacional & & & .000 & .002 & -.034 & .973 \\
\hline Competencia & & & .003 & .004 & .676 & .502 \\
\hline
\end{tabular}

${ }^{*} p<.10$ 
En términos generales, el análisis de regresión mostró que las variables orientación empresarial, esfuerzo de aprendizaje organizacional y competencia no reflejaron relación estadísticamente significativa (a un nivel máximo de significancia de .10) con la internacionalización de las empresas de la muestra. Sólo la variable ambiente mostró relación estadísticamente significativa (a un nivel de significancia de .10), pero reflejó un coeficiente que indicaba una relación negativa. Esto no proveyó apoyo a las hipótesis del estudio $\left(\mathrm{H}_{1}, \mathrm{H}_{2}\right.$, $\mathrm{H}_{3}, \mathrm{H}_{4}$ ) que, como se señaló anteriormente, planteaban relaciones positivas significativas de las cuatro variables independientes con respecto a la variable dependiente.

Además de analizar las variables independientes identificadas con respecto a la internacionalización, también se realizó un análisis de regresión múltiple de las variables demográficas con respecto a las ventas internacionales (véase Tabla 3). Se especificó un modelo (ecuación de regresión) donde la variable dependiente ventas internacionales (INTSALES) será una función de las variables independientes (años operando (YBUSS), años en el comercio internacional (YINTB), cantidad de empleados (EMPLOYEES), ventas (SALES), cantidad o número de países a los que exporta (COUNTRYEXP) y frecuencia con la que viajan los ejecutivos de la empresa (TRAVEL) más un término de error, como se establece en la siguiente ecuación:

$$
\begin{aligned}
\text { INTSALES }= & \beta_{0}+\beta_{1} \text { YBUSS }+\beta_{2} \text { YINTB }+\beta_{3} \text { EMPLOYEES }+\beta_{4} \\
& \text { SALES }+\beta_{5} \text { COUNTRYEXP }+\beta_{6} \text { TRAVEL }+ \text { Error }
\end{aligned}
$$

En la Tabla 3 se presenta un resumen de los resultados del análisis de regresión utilizando la ecuación antes expuesta. 
Tabla 3: Resumen del análisis de regresión de los datos demográficos contra ventas internacionales

INTSALES $=\beta_{0}+\beta_{1}$ YBUSS $+\beta_{2}$ YINTB $+\beta_{3}$ EMPLOYEES $+\beta_{4}$ SALES $+\beta_{5}$ COUNTRYEXP $+\beta_{6}$ TRAVEL + Error

\begin{tabular}{|c|c|c|c|c|c|c|}
\hline $\begin{array}{l}\text { Variable } \\
\text { independiente }\end{array}$ & $\begin{array}{l}\text { Variable } \\
\text { dependiente }\end{array}$ & $\mathrm{R}^{2}$ & $\beta$ & $\begin{array}{l}\text { Error } \\
\text { estándar }\end{array}$ & t-value & $p$-value \\
\hline Constante & $\begin{array}{l}\text { Ventas } \\
\text { internacionales }\end{array}$ & .436 & .007 & .069 & .099 & .921 \\
\hline $\begin{array}{l}\text { Años de } \\
\text { operación }\end{array}$ & & & -.005 & .002 & -2.075 & $.042^{* *}$ \\
\hline $\begin{array}{l}\text { Años en el } \\
\text { comercio } \\
\text { internacional }\end{array}$ & & & .005 & .005 & .901 & .371 \\
\hline $\begin{array}{l}\text { Número de } \\
\text { empleados }\end{array}$ & & & .006 & .032 & .182 & 856 \\
\hline Ventas totales & & & .002 & .010 & .220 & .827 \\
\hline $\begin{array}{l}\text { Número de países } \\
\text { a los que exporta }\end{array}$ & & & .024 & .007 & 3.393 & $.001^{* * *}$ \\
\hline $\begin{array}{l}\text { Frecuencia de } \\
\text { viajes al año }\end{array}$ & & & .033 & .013 & 2.452 & $.017^{* *}$ \\
\hline $\begin{array}{l}\text { Regiones } \\
\text { destinario de las } \\
\text { exportaciones }\end{array}$ & & & .026 & .015 & 1.682 & .973 \\
\hline
\end{tabular}

${ }^{*} p<.10 .{ }^{* *} p<.05 .{ }^{* * *} p<.01$

De acuerdo con los resultados, las variables años de operación, número de países a los que se exporta, frecuencia de viajes al año y regiones destinatarias de las exportaciones mostraron relación a diferentes niveles de significancia. Los años de operación de las empresas mostró una relación negativa con ventas internacionales, lo que sugiere que mientras más antiguas las empresas, menores son sus ventas internacionales como por ciento de sus ventas totales. El número de países a los que se exporta mostró una relación positiva, lo cual se interpreta que cuanto mayor sea el número de países ma- 
yor las ventas internacionales. La frecuencia de viajes al año de los ejecutivos mostró una relación positiva con el nivel de ventas internacionales. Las regiones destinatarias de las exportaciones presentaron una relación positiva, pero a un nivel de significancia menor que las anteriores variables demográficas. ${ }^{1}$

\section{Discusıón}

La falta de relación entre la orientación empresarial y la internacionalización en nuestro estudio contrasta con hallazgos en un estudio cuantitativo reciente entre 197 PYMES (la mayoría de manufactura) en la costa este de Estados Unidos, el cual encontró relación positiva entre orientación empresarial e internacionalización (Todd, 2006). Los hallazgos de dicho estudio resultan una buena comparación debido a que midió orientación empresarial unidimensionalmente con la misma escala (compuesto por los mismos ítems) utilizada en el presente estudio. En un estudio entre 32 PYMES de manufactura en el área de Nueva Inglaterra (Estados Unidos), Olsen-Waldron (2004) encontró que las dimensiones o subescalas de la orientación empresarial varían en sus interrelaciones con el nivel de internacionalización de las empresas. Sus hallazgos respaldan los hallazgos de Lumpkin y Dess (1997) quienes encontraron que la orientación empresarial es una variable multidimensional, cuyas relaciones con otras variables pueden variar dependiendo del contexto comercial o de negocios en que se evalúen. Considerando esto, el presente estudio tampoco encontró relación estadísticamente significativa entre cada subdimensión de la orientación empresarial (proactividad, innovación y toma de riesgos) y las ventas internacionales.

Nuestro análisis de regresión tampoco encontró relación estadísticamente significativa entre el esfuerzo de aprendizaje organizacional y la internacionalización de las PYMES. Esto contrasta con

\footnotetext{
${ }^{1}$ Para el análisis de regresión, las regiones destinatarias de las exportaciones fueron codificadas asignando un peso que representaban la distancia geográfica y cultural con respecto al mercado nacional de las empresas. Por ejemplo, se le asignó un peso de 1 a Norteamérica (Estados Unidos, México y Canadá), 2 a los países de la región del Caribe, 3 a los países de Centroamérica, 4 a los países de Suramérica, 5 a los países europeos y 6 a los países asiáticos.
} 
los hallazgos de De Clercq et al., (2005) quienes reportaron una relación positiva estadísticamente significativa entre el esfuerzo de aprendizaje internacional y la intención de internacionalización entre 92 PYMES de siete industrias diferentes en Bélgica. Cabe señalar que el estudio de De Clercq utilizó la variable dependiente intención de internacionalización como una multidimensional que incluía tres dimensiones: las ventas extranjeras como por ciento de las ventas totales; el por ciento de empleados que invertían tiempo en actividades internacionales de la empresa; y el alcance geográfico de las ventas internacionales.

Autio et al. (2000) reportaron una relación positiva estadísticamente significativa entre intensidad de conocimiento y crecimiento en ventas internacionales entre 77 PYMES en la industria electrónica en Finlandia. Más recientemente, Basly (2007) encontró una relación positiva entre el conocimiento de internacionalización y el grado de internacionalización entre 118 empresas familiares de varias industrias en Finlandia. Estos resultados contrastan con el presente estudio, que no encontró relación entre los esfuerzos de aprendizaje con propósito de explotar y renovar conocimiento con respecto a las actividades internacionales (y al mercado exterior más importante) con las ventas internacionales.

Contrario a lo planteado en la hipótesis de trabajo, el presente estudio encontró que la variable ambiente tiene relación negativa estadísticamente significativa con la internacionalización de las PYMES en Puerto Rico. Entre 134 empresas de manufactura en Estados Unidos, Zahra (1993) encontró una relación positiva entre dinamismo de ambiente y el desempeño financiero, resultante de la introducción de productos nuevos. Otro estudio sobre PYMES de manufactura encontró que cuando la hostilidad del mercado nacional es alta, las empresas utilizan modos de entrada a mercados extranjeros no basados en capital (i.e., exportación) y tienen una tasa mayor de crecimiento de ingresos internacionales (Rasheed, 2005). A pesar de estos estudios, también se ha encontrado relación negativa entre la hostilidad del ambiente y la orientación empresarial, lo que sugiere que las empresas o los gerentes tienden a ser reactivos a medida que perciben un aumento en la hostilidad del ambiente, y proactivos, innovadores y arriesgados cuando perciben que el 
ambiente se vuelve más benigno (Miles et al., 1993). Dicho hallazgo se alinea con los resultados de nuestro estudio, que a mayor incertidumbre de ambiente, menor internacionalización de las PYMES en Puerto Rico.

El presente estudio tampoco encontró relación entre la intensidad de la competencia y la internacionalización de la PYMES. La competencia es una variable que influye en la internacionalización de las empresas (Cullen \& Parboteeah, 2005; Czinkota, Ronkainen \& Moffett, 2002). Estudios sobre PYMES estadounidenses con instalaciones operacionales en Europa han señalado el aumento en la competencia en el mercado nacional como uno de los conductores (aunque no es el más importante o el mencionado con mayor frecuencia) para la expansión a mercados extranjeros (Prater \& Ghosh, 2005). La competencia se ha señalado como una fuerza motivadora que influye en la rapidez con la que una empresa se internacionaliza (Oviatt \& McDougall, 2005). De hecho, estudios de caso en Puerto Rico han identificado y señalado las presiones de la competencia como una de las variables que ha llevado a empresas puertorriqueñas a establecer subsidiarias de producción en países de la región del Caribe cercanos a Puerto Rico (Orengo-Serra, 2007). Sin embargo, estudios cuantitativos no han encontrado efecto moderador estadísticamente significativo de la intensidad de la competencia con respecto a la orientación de mercado que asume una empresa y su desempeño (Jaworski \& Kohli, 1993). Dichos hallazgos se alinean con los resultados del presente estudio cuantitativo sobre las PYMES en Puerto Rico que exportan su producto y/o servicio.

Cabe señalar que entre las variables o datos demográficos se encontró una relación positiva estadísticamente significativa (a diferentes niveles de significancia) del número de países a los que se exporta, las regiones geográficas destinatarias de las exportaciones y la frecuencia de viajes con respecto a las ventas internacionales de las empresas encuestadas. Dichos resultados se alinean con los planteamientos y hallazgos de estudios con respecto a que la internacionalización de las PYMES se da por etapas (Dollinger, 1995).

$\mathrm{Al}$ analizar las estadísticas descriptivas de las variables demográficas de las empresas y los dueños o gerentes de ventas internacionales encuestados, encontramos que las PYMES en Puerto Rico 
que exportan cuentan con al menos 20 años de operaciones, tienen menos de 75 empleados y sus ingresos anuales pueden fluctuar desde menos de $\$ 500,000$ hasta más de $\$ 10$ millones. Las empresas presentaron un promedio de 9 años en el comercio internacional, con unas ventas internacionales que representan aproximadamente el $24 \%$ de las ventas totales. No obstante, también se encontraron dos empresas que en los primeros cinco años de establecidas reportan que sus ventas internacionales representan más del $75 \%$ de las ventas totales, proporciones de años de operación y ventas internacionales que cumplen con el fenómeno identificado en la literatura académica como empresas "born global" (se encontraron tres empresas más que reportaron ventas internacionales de entre $50 \%$ al $74 \%$ con solamente dos y seis años de establecidas).

Debemos resaltar que el $90 \%$ de las empresas encuestadas identificaron la exportación como su estrategia o método para entrar y atender su mercado extranjero. En promedio, las empresas encuestadas informaron que exportaban a seis países distintos. Aunque las empresas señalaron exportar a países tan lejanos como Japón, la mayor parte exportaba a Estados Unidos y las islas del Caribe (considerando cada isla del Caribe como un país de exportación diferente).

Los dueños o gerentes de ventas internacionales encuestados promediaron 42 años de edad, 14 años de experiencia en la industria que trabajaban actualmente y 10 años de experiencia en el comercio internacional. Aproximadamente, el 90\% tenía al menos un grado de bachillerato universitario (casi un 39\% contaban con posgrados) y todos señalaron hablar y/o leer inglés. Unos pocos expresaron hablar francés, portugués e italiano. Un poco más de una cuarta parte de las personas encuestadas indicó que la mayor parte de su conocimiento en el área de comercio internacional ha sido de carácter autodidáctico. Esto muchas veces resulta en procesos trialerror que pueden atrasar el éxito de la internacionalización o incluso puede desincentivar la iniciativa de exportar productos a mercados extranjeros. Se ha reportado que los obstáculos relacionados con la exportación se pueden resumir en la falta de contactos comerciales (el asunto de la distribución es de preocupación especial), conocimiento de los mercados extranjeros, aspectos técnicos de los métodos de pago y falta de tiempo y dinero (Carrier, 1999). Esto es un 
punto para consideraciones de políticas e iniciativas del gobierno con respecto a programas de orientación.

\section{Conclusión}

En estudios de casos sobre PYMES puertorriqueñas se ha documentado aspectos propios de una orientación empresarial estratégica como motivadores o factores influyentes en la internacionalización de éstas (Orengo-Serra, 2007). Sin embargo, en una muestra de 71 empresas, no hubo evidencia de que la orientación empresarial refleje un punto de vista estratégico relacionado con las ventas internacionales. En otras palabras, las ventas internacionales pueden no depender de que una empresa se mueva proactivamente en nuevos mercados y adopte un punto de vista innovador y de toma de riesgo, puesto que éstas pueden ser dirigidas primeramente para su mercado nacional. Esto sugiere que la orientación empresarial de las empresas encuestadas resulta en un punto de vista estratégico dirigido primeramente al mercado nacional, por lo que no se refleja en las ventas internacionales.

La falta de relación entre los esfuerzos de aprendizaje organizacional y la internacionalización de las empresas encuestadas sugiere que aunque se hayan realizado los esfuerzos, éstos no se han traducido en mayores ventas internacionales. Tanto la orientación empresarial como los esfuerzos de aprendizaje organizacional son considerados e identificados como factores internos de la empresa. Entonces, se puede concluir que la internacionalización de las PYMES en Puerto Rico al momento del estudio no está relacionada con factores propios de las empresas.

Como factores externos de las empresas, este estudio consideró el ambiente y la competencia. A diferencia de lo identificado en otros estudios de casos, la competencia o la intensidad competitiva no mostró relación con las ventas internacionales de las PYMES encuestadas. Esto indica que el nivel de competencia que enfrentan las PYMES que exportan no repercute en sus ventas internacionales. Por su parte, el ambiente (medido por niveles de dinamismo, hostilidad y heterogeneidad) mostró una relación negativa con las ventas internacionales de las PYMES encuestadas. Esto indica que 
a mayor dinamismo, hostilidad y heterogeneidad (complejidad) del ambiente menor las ventas internacionales. Esto sugiere que un ambiente nacional con menos dinamismo y hostilidad influye, o, al menos, aumenta, la propensión de las PYMES exportadoras de Puerto Rico a aumentar sus ventas internacionales. Estadísticamente, el ambiente es la variable que más poder explicativo presenta sobre la internacionalización de las PYMES en Puerto Rico. Sin embargo, la falta de relación de las otras variables consideradas con respecto a la internacionalización de las PYMES en Puerto Rico también aporta explicaciones cualitativas.

La falta de relación de orientación empresarial y la relación negativa de ambiente con respecto a las ventas internacionales reflejan que las PYMES exportadoras en Puerto Rico son empresas tradicionales que presentan una orientación empresarial dirigida primordialmente a la estabilidad y solidez en el mercado nacional para luego considerar exportar. En otras palabras, ante la hostilidad del ambiente como son el aumento en el salario mínimo, costos de transportación y costos operacionales (e.g., servicio eléctrico), las empresas dan prioridad a ajustes en sus operaciones nacionales (donde se encuentra su mayor ingreso), que pueden redundar en una disminución de los recursos económicos y humanos destinados a atender el mercado extranjero, y que a su vez lleva a una disminución en las ventas internacionales de la empresa. Por su parte, la falta de relación de esfuerzos de aprendizaje organizacional internacional y la internacionalización de las PYMES en Puerto Rico, sugiere una falta de estrategia o de planificación estratégica que lleve el conocimiento a un resultado en las ventas internacionales. No obstante, se debe mencionar que el ambiente nacional influye en el punto de vista estratégico de las PYMES. Matthews y Scott (1995) han encontrado que la planificación estratégica formal entre las PYMES es inversamente proporcional a la incertidumbre en sus ambientes comerciales. Cuando la incertidumbre en el ambiente es alta, las PYMES tienden a fiarse más en "instintos" que en un proceso de planificación formal, lo que puede llevar a unos "ajustes instintivos" en las operaciones generales de la empresa que resulten en una disminución de las ventas internacionales. Tal vez la falta de estrategia de las PYMES en Puerto Rico en sus actividades internacionales sea la causa de que 
las variables independientes consideradas no son las más predictivas de la internacionalización de las PYMES en Puerto Rico.

\section{Contribución e IMPLICACIONES}

Los estudios recientes sobre la internacionalización de las PYMES en Puerto Rico se han limitado a casos de estudios particulares de empresas que han mostrado una internacionalización consistente a través de los años (Orengo-Serra, 2007). El presente estudio cuantitativo que abarca empresas de diversas industrias (manufactura, servicio y mayoristas/minoristas) proporciona información sobre características o rasgos generales de la internacionalización de las PYMES en Puerto Rico. Esto permite identificar problemas o prácticas comunes que tienen implicaciones o consideraciones gerenciales teóricas y prácticas y así como de política pública para el gobierno de Puerto Rico.

Estudios entre PYMES en países como Bélgica y Finlandia, han encontrado relación positiva entre la orientación empresarial, los esfuerzos de aprendizaje organizacional y las ventas internacionales (Autio et al., 2000; De Clercq et al., 2005). Estos países, al igual que Puerto Rico, representan países pequeños cuyos nacionales domésticos son limitados. Estos hallazgos nos llevan a la función del Gobierno como agente intermediario no sólo para promover entre la clase empresarial puertorriqueña la conciencia de la importancia o el impacto de desarrollar un conocimiento internacional a través de las organizaciones, sino también para proveer herramientas que permitan una estrategia de internacionalización y su posterior ejecución efectiva. Todavía una cuarta parte (28\%) de los dueños, ejecutivos o personal gerencial de las empresas encuestadas señala que el mayor conocimiento de comercio internacional que tiene es resultado de gestiones propias. Dado que la población del estudio provino de una lista de una agencia del gobierno de Puerto Rico que ayuda a las empresas puertorriqueñas en el proceso de internacionalizarse, lo anterior es indicativo de que todavía las agencias de gobierno tienen trabajo que hacer.

El presente estudio presenta un escenario de la internacionalización de las PYMES en Puerto Rico. La presencia o ausencia de 
relaciones entre las variables independientes (identificadas como factores internos y externos) y la internacionalización (ventas internacionales) puede presentar una guía para los empresarios y los que crean política pública en términos de dónde dirigir recursos y esfuerzos cuando se considera las PYMES y su potencial crecimiento a través de una expansión a mercados extranjeros. Este trabajo sirve como marco de referencias o punto de partida para futuras investigaciones que escudriñen la internacionalización de las PYMES en Puerto Rico, con el propósito de desarrollar el potencial de internacionalización de una clase empresarial puertorriqueña educada. 


\section{REFERENCIAS}

Andersen, O. (1993). On the internationalization process of firms: A critical analysis. Journal of International Business Studies, 24(2), 209-231.

Andersson, S. (2000). The internationalization of the firm from an entrepreneurial perspective. International Studies of Management E Organization, 30(1), 63-92.

Andersson, S., Gabrielsson, J., \& Wictor, I. (2004). International activities in small firms: Examining factors influencing the internationalization and export growth of small firms. Canadian Journal of Administrative Sciences, 21(1), 22-34.

Andersson, S., \& Wictor, I. (2003). Innovative internationalization in new firms: Born globals-the Swedish Case. Journal of International Entrepreneurship, 1(3), 249-276.

Autio, E., Sapienza, H., \& Almeida, J. (2000). Effect of age at entry, knowledge intensity, and imitability on international growth. Academy of Management Journal, 43(5), 909-924.

Basly, S. (2007). The internationalization of Family SME: An organizational learning and knowledge development perspective. Baltic Journal of Management, 2(2), 154-180.

Bosma, N., Jones, K., Aution, E., \& Levie, J. (2007). Global Entrepreneurship Monitor 2007 Executive Report. Global Entrepreneurship Research Association.

Carrier, C. (1999). The training and development needs of owner-manager of small business with export potential. Journal of Small Business Management, 37(4), 30-41.

Cateora, P. R., \& Graham, J. (2007). International Marketing. $13^{\text {th }}$ Edition. New York: McGraw-Hill Irwin.

Chetty, S., \& Campbell-Hunt, C. (2004). A strategic approach to internationalization: A traditional versus "born-global". Journal of International Marketing, 12(1), 57-81.

Compañía de Comercio y Exportación de Puerto Rico. (2007). Puerto Rico Export Catalogue E Interactive Directory. Accedido el 9 de enero de 2008 de http://www.comercioyexportacion.com/comercioyexportacion/PREC_2/default.htm 
Coviello, N. E., \& McAuley, A. (1999). Internationalisation and the smaller firm: A review of contemporary empirical research. Management International Review, 39(3), 223-256.

Coviello, N. E., \& Munro, H. J. (1995). Growing the entrepreneurial firm: Networking for international market development. European Journal of Marketing, 29(7), 49-61.

Covin, J. G., \& Slevin, D. P. (1991). A conceptual model of entrepreneurship as firm behavior. Entrepreneurship: Theory $\mathcal{E}$ Practice, 16(1), 7-25.

Covin, J. G., \& Slevin, D. P. (1989). Strategic management of small firms in hostile and benign enviroments. Strategic Management Journal, 10(1), 75-87.

Cullen, J. B., \& Praveen Parboteeah, K. (2005). Multinational Management: A Strategic Approach. $3^{\text {rd }}$ Edition. Ohio: South-Western, Thomson Learning.

Czinkota, M., Ronkainen, I. A., \& Moffett, M. (2002). International Business. $6^{\text {th }}$ Edition. Ohio: South-Western College Publishing.

David, F. (1999). Strategic Management: Concepts and Cases. $12^{\text {th }}$ Edition. New Jersey: Prentice Hall, Inc.

De Clercq, D., Sapienza, H. J., \& Crijns, H. (2005). The internationalization of small and medium-sized firms. Small Business Economics 24(4), 409-419.

Dess, G. G., \& Beard, D. W. (1984). Dimensions of organizational task environments. Administrative Science Quarterly, 29(1), 52-73.

Dodge, H. R., Fullerton, S., \& Robbins, J. E. (1994). Stage of the organizational life cycle and competition as mediators of problem perception for small business. Strategic Management Journal, 15(2), 121-134.

Dollinger, M. (1995). Entrepreneurship. Burr Ridge, Illinois: Irwin.

Etemad, H. (2004). Internationalization of small and medium-sized enterprises: A grounded theoretical framework and an overview. Canadian Journal of Administrative Sciences, 21(1), 1-21.

French, M. (2006). Export Market Orientation: Performance, Timing, and Extent of Internationalization. Disertación Doctoral, Nova Southeastern University.

Hair, J. F., Anderson, R. E., Tatham, R. L. \& Black, W. C. (1998). Multivariate Data Analysis. $5^{\text {th }}$ Edition. New Jersey: Prentice Hall. 
Hill, C. W. (2003). International Business: Competing in the Global Marketplace. $4^{\text {th }}$ Edition. New York: McGraw-Hill Irwin.

Jaworski, B. J., \& Kohli, A. K. (1993). Market orientation: Antecedents and consequences. Journal of Marketing, 57(3), 53-70.

Johanson, J., \& Vahlne, J. E. (1990). The mechanism of internationalization. International Marketing Review, 7(4), 11-24.

Keegan, W. (1999). Global Marketing Management. $6^{\text {th }}$ Edition. New Jersey: Prentice Hall.

Lumpkin, G. T., \& Dess, G. G. (1997). Proactiveness versus competitive aggressiveness: Teasing apart key dimension of an entrepreneurial orientation. Frontiers of Entrepreneurship Research. Wellesley, MA: Babson College.

Matthews, C. H., \& Scott, S. G. (1995). Uncertainty and planning in small and entrepreneurial firms: An empirical assessment. Journal of Small Business Management, 33(4), 34-52.

Miles, M. P., Arnold, D. R., \& Thompson, D. L. (1993). The interrelationship between environmental hostility and entrepreneurial orientation. Journal of Applied Business Research, 9(4), 12-24.

Miller, D., \& Friesen, P. H. (1982). Innovation in conservative and entrepreneurial firms: Two models of strategic momentum. Strategic Management Journal, 3(1), 1-25.

Menguzzato-Boulard, M., \& Sanchez-Peinado, L. (2007). Entrepreneurial orientation and international commitment. Journal of International Entrepreneurship, 5(3/4), 65-83.

Moen, Ø., \& Servais, P. (2002). Born global or gradual global? Examining the export behavior of small and medium-sized enterprises. Journal of International Marketing, 10(3), 49-72.

Musteen, M. (2006). The Role of International Networks and Foreign Market Knowledge in the Internationalization of Czech Entrepreneurial Ventures. Disertación Doctoral, University of Kansas.

Nunnally, J. C. (1978). Psychometric Theory. $2^{\text {nd }}$ Edition. New York: McGraw Hill.

Ogunmokun, G., \& Ng, S. (2004). Factors influencing export performance in international marketing: A study of Australian firms. International Journal of Management, 21(2), 172-185. 
Olsen-Waldron, Nancy. (2004). Entrepreneurial Orientation and its relation to the Internationalization of Small Business Manufacturing Enterprises in New England. Disertación Doctoral no publicada, Capella University.

Orengo-Serra, K. (2007). Empresas puertorriqueñas en la hora de la integración regional. Massachussets: Cambridge BrickHouse Inc.

Organisation for Economic Cooperation and Development. (2005). SME and Entrepreneurship Outlook, 2005. Paris: OECD Publication.

Oviatt, B. M., \& McDougall, P. P. (2005). Defining international entrepreneurship and modeling the speed of internationalization. Entrepreneurship Theory and Practice, 29(5), 537-553.

Oviatt, B. M., \& McDougall, P. P. (1997). Challenges for internationalization process theory: The case of international new ventures. Management International Review, 37(2), 85-100.

Prater, E., \& Ghosh, S. (2005). Current operational practices of U.S. small and medium-sized enterprises in Europe. Journal of Small Business Management 43(2), 155-169.

Rasheed, H. S. (2005). Foreign entry mode and performance: The moderating effects of environment. Journal of Small Business Management, 43(1), 41-54.

Rennie, M. W. (1993). Born global. McKinsey Quarterly, 4, 45-52.

Rialp, A., Rialp, J., Urbano, D., \& Vaillant, Y. (2005). The born-global phenomenon: A comparative case study research. Journal of International Entrepreneurship, 3(2), 133-171.

Rodrigues, C. (2001). International Management: A Cultural Approach. $2^{\text {nd }}$ Edition. Cincinnati, Ohio: South Western College Publishing, Thompson Learning.

Ruzzier, M., Hisrich, R. D., \& Antoncic, B. (2006). SME internationalization research: Past, present, and future. Journal of Small Business and Enterprise Development, 13(4), 476-497.

Todd, P. (2006). An Empirical Investigation of Entrepreneurial Orientation, Internationalization, and Performance of Small and Medium-sized Enterprise. Disertación Doctoral, Cleveland State University.

Wolff, J. A., \& Pett, T. L. (2000). Internationalization of small firms: An examination of export competitive patterns, firms size, and export performance. Journal of Small Business Management, 38(2), 34-47. 
Zahra, S. A. (1993). New product innovation in established companies: Associations with industry and strategy variables. Entrepreneurship: Theory E Practice, 18(2), 47-69. 